Am J Nephrol 2021;52:714-724

DOI: $10.1159 / 000518455$
Received: May 13, 2021

Accepted: July 12, 2021

Published online: September 8, 2021

\title{
Urinary Plasminogen Activator Inhibitor-1: A Biomarker of Acute Tubular Injury
}

\author{
María Paniagua-Sancho a,b,c Yaremi Quirosa, c, d Alfredo G. Casanovaa, b, c \\ Víctor Blanco-Gozalo a, b, c Consuelo Agüeros-Blanco ${ }^{\mathrm{e}}$ \\ Adalberto Benito-Hernández ${ }^{e}$ María A. Ramos-Barron ${ }^{e}$

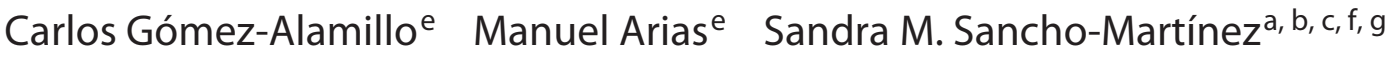 \\ Francisco J. López-Hernández ${ }^{a}$ b, c, d, f, g \\ anstitute of Biomedical Research of Salamanca (IBSAL), Salamanca, Spain; bepartment of Physiology and \\ Pharmacology, University of Salamanca, Salamanca, Spain; 'Group of Translational Research on Renal and \\ Cardiovascular Diseases (TRECARD), Salamanca, Spain; ${ }^{\mathrm{d}}$ Instituto de Estudios de Ciencias de la Salud de Castilla y \\ León (IECSCYL), Soria, Spain; ${ }^{e}$ Department of Nephrology, Hospital Universitario Marqués de Valdecilla, Instituto \\ de Investigación Sanitaria Valdecilla (IDIVAL), Santander, Spain; ${ }^{\mathfrak{f} G r o u p ~ o f ~ B i o m e d i c a l ~ R e s e a r c h ~ o n ~ C r i t i c a l ~ C a r e ~}$ \\ (BioCritic), Valladolid, Spain; 9Spanish Renal Research Network (REDinREN), Instituto de Salud Carlos III, Madrid, Spain
}

\section{Keywords}

Acute kidney injury · Acute tubular necrosis · PAI-1 · Urinary biomarker - Tubular injury

\begin{abstract}
Introduction: Acute kidney injury (AKI) is a threatening, multiaetiological syndrome encompassing a variety of forms and damage patterns. AKI lacks sufficiently specific diagnostic tools to evaluate the distinct combination of pathophysiological events underlying each case, which limits personalized and optimized handling. Therefore, a pathophysiological diagnosis based on new urinary biomarkers is sought for practical (readiness and noninvasiveness) and conceptual reasons, as the urine is a direct product of the kidneys. However, biomarkers found in the urine may also have extrarenal origin, thus conveying pathophysiological information from other organs or tissues. Urinary plasminogen activator inhibitor-1 (PAI-1) has been associated to
\end{abstract}

AKI, although its origin and traffic to the urine are not known. Methods: Herein, we studied the blood or renal origin of urinary PAl-1 (UPAI-1) in experimental AKI in Wistar rats, by means of the in situ renal perfusion method. For this purpose, urine was collected while the kidneys of rats with AKI showing increased UPAI1 excretion, and controls, were in situ perfused with a saline solution. Results: Our results show that during perfusion, PAl-1 remained in the urine of AKI rats, suggesting that renal cells shed this protein directly to the urine. PAI- 1 is also significantly increased in the urine of AKI patients. Its low correlation with other urinary markers such as NGAL or NAG suggests that PAI-1 provides complementary and distinct phenotypical information. Conclusion: In conclusion, uPAI-1 is a biomarker produced by damaged kidneys following AKI, whose precise pathophysiological meaning in AKI needs to be further investigated.

(c) 2021 S. Karger AG, Basel

María Paniagua-Sancho and Yaremi Quiros share first authorship. 


\section{Introduction}

Acute kidney injury (AKI) involves a sudden loss of renal excretory function and is defined according to internationally recognized scales on increments in plasma creatinine concentration $\left(\mathrm{Cr}_{\mathrm{pl}}\right)$ or reductions in urinary output [1]. In many cases, after an AKI episode renal function returns apparently to normal parameters in a few days. However, in other cases, renal function and renal tissue structure undergo a variety of deleterious consequences, ranging from delayed recovery to the need of renal replacement therapy (i.e., dialysis) for life, or even death [2]. Worst scenarios are frequently observed among critically ill patients and in the context of intensive medicine [2]. In all cases, including mild and reversible, and even subclinical episodes, increased morbidity and mortality and incidence of chronic kidney disease are observed after AKI [2]. Prognosis of AKI is still difficult to estimate, beyond the assumption that milder cases tend to show a better prognosis, and that prerenal (i.e., hemodynamic) AKI is associated with milder outcomes than intrinsic AKI, such as acute tubular injury (ATI), formerly known as acute tubular necrosis $[3,4]$. Thus, a precise pathophysiological diagnosis is necessary to study and then associate specific injury patterns to specific outcomes [5].

Attainment of this unmet need is limited by the lack of aetiopathological biomarkers, whose elevations (or decrements) in bodily samples be known to derive from concrete pathophysiological events. In this sense, the gold standard biomarker (i.e., $\mathrm{Cr}_{\mathrm{pl}}$ ) increases irrespective of cause or underlying process. Last generation markers erupting into the clinical practice, such as neutrophil gelatinase-associated lipocalin (NGAL), kidney injury molecule 1 (KIM-1), tissue inhibitor of metalloproteinase 2 (TIMP-2), and insulin-like growth factor-binding protein 7 (IGFBP7), incorporate some degree of specificity and sensitivity to AKI diagnosis beyond $\mathrm{Cr}_{\mathrm{pl}}$ [6-8]. Most, if not all of these markers have been long believed to be produced by damaged kidneys (i.e., by tubular epithelial cells) and directly shed to the urine, where they serve as biomarkers of tubular damage. However, this concept has been questioned recently. It has been shown that the increased urinary level of NGAL $[9,10]$ and TIMP-2 and IGFBP7 [11] is not the result of direct shedding from tubule cells, but of reduced tubular reclamation of the filtered proteins. These findings might modify the pathophysiological and diagnostic meaning of these biomarkers.

New markers, with distinct biological meaning or sensitivity for individual biological processes, are thus needed to build fingerprints of aetiopathological varieties of
AKI, which can be used for pathophysiological diagnosis and thereof associated to distinctive prognosis. Plasminogen activator inhibitor-1 (PAI-1) is an endogenous inhibitor of thrombolysis with pro-coagulant properties. Tissue and plasma levels of PAI-1 increase in coagulation, fibrotic and inflammatory disorders [12]. We previously reported abnormally elevated levels of PAI-1 in the urine of rats undergoing nephrotoxic AKI $[13,14]$. However, its role in this type of AKI and, more specifically, the meaning of urinary PAI-1 (uPAI-1) in AKI diagnosis has not been thoroughly explored. In this article, we studied the urinary excretion of UPAI-1 in diverse animal models and patients of AKI, and deepened on its origin and traffic, with the aim of further understanding its diagnostic significance and utility.

\section{Materials and Methods}

Reagents were purchased from Sigma (Madrid, Spain), except where otherwise indicated.

Animal Models and Experimental Protocols

All procedures were approved by the Bioethics Committee of the University of Salamanca. Animals were handled according to the guidelines of the European Community Council Directive 2010/63/UE, and to the current Spanish legislation for experimental animal use and care, RD 53/2013. Three rat models of ATI, a most common form of intrinsic AKI, were used to study uPAI-1 excretion under typical aetiopathological scenarios involving ischemic and nephrotoxic acute renal damage. Wistar rats (220-240 g) were housed under controlled experimental conditions and allowed free access to regular chow and water. Rats were divided into 5 groups ( $n=6$ per group): (1) control group (Control), saline solution; (2) ischemia-reperfusion group (I/R), $1 \mathrm{~h}$ of warm renal ischemia plus contralateral nephrectomy; (3) gentamicin group $(G)$, receiving gentamicin i.p. (150 mg/kg per day) for 6 days; (4) cisplatin group (CDDP), a single i.p. administration of $5 \mathrm{mg} / \mathrm{kg}$ cisplatin.

At specific time points, rats were allocated in metabolic cages to obtain 24-h, individual urine samples. Blood was drawn with a needle from the tail vein, and plasma was obtained by centrifugation and, at the end of the treatments, kidneys were perfused with heparinized saline and immediately dissected. Half of the kidney was fixed in paraformaldehyde for histological studies, and the rest was frozen at $-80^{\circ} \mathrm{C}$ for biochemical analysis.

Urinary Excretion Studies with in situ Perfused Kidneys

At the end of the experimental protocol or treatment (i.e., day 1 in the I/R group; day 6 in the G group; and day 4 in the CDDP group), some rats were anesthetized ( $50 \mathrm{mg} / \mathrm{kg}$ sodium pentobarbital) and an extracorporeal circuit for kidney perfusion was set up, as described elsewhere [9]. Briefly, the renal artery, vein, and ureter of the right kidney were ligated. The renal artery and vein of the left kidney and the urinary bladder were cannulated. Oxygenated and warm $\left(37^{\circ} \mathrm{C}\right)$ Krebs-dextran $(40 \mathrm{~g} / \mathrm{L}$ dextran [molecular weight $64 \mathrm{k}-76 \mathrm{k}$ ] in Krebs solution [120 mM NaCl, $4.8 \mathrm{mM} \mathrm{KCl}, 1.8 \mathrm{mM} \mathrm{CaCl}_{2}$, 
$1.2 \mathrm{mM} \mathrm{MgSO}_{4}, 1.2 \mathrm{mM} \mathrm{KH}_{2} \mathrm{PO}_{4}, 25 \mathrm{mM} \mathrm{NaHCO} 3,0.026 \mathrm{~mm}$ EDTA, 11.1 glucose, $\mathrm{pH}=7.4]$ ) was perfused through the renal artery at $3 \mathrm{~mL} / \mathrm{min}$. The effluent from the renal vein was collected during $60 \mathrm{~min}$ of perfusion or was discarded. Urine fractions were collected (every $20 \mathrm{~min}$ ) from a catheter placed in the urinary bladder, starting $20 \mathrm{~min}$ before the perfusion with Krebs (when blood was still passing through the kidney), and during 60 min after perfusion with Krebs started.

\section{Renal Function}

Renal function was monitored by means of $\mathrm{Cr}_{\mathrm{pl}}$ and urea and urinary protein concentration. $\mathrm{Cr}_{\mathrm{pl}}$ and urea were determined with commercial colorimetric kits (Quantichrom Creatinine and Quantichrom Urea Assay Kits; BioAssay Systems, Hayward, CA, USA), according to the manufacturer's instructions. Proteinuria was determined with a commercial kit (Bio-Rad, Madrid, Spain) based in the Lowry method.

\section{Renal Histopathology}

Paraformaldehyde-fixed rat tissue samples were immersed in paraffin, cut into $2 \mu \mathrm{m}$-thick slices, and stained with hematoxylin and eosin. Renal specimens were then photographed under an Olympus BX51 microscope connected to an Olympus DP70 color, digital camera (Olympus, Madrid, Spain).

\section{Patients and Clinical Protocol}

Urine samples were collected from 72 volunteers from the $\mathrm{Ne}$ phrology Department (Hospital Universitario Marqués de Valdecilla, Santander, Spain), who provided written consent: 63 consultation patients referred to Nephrology had AKI at admission; and 9 controls. All protocols were approved by the local Ethics Committee and were conducted according to the principles established in the Declaration of Helsinki (World Medical Assembly), the Council of Europe Convention on Human Rights and Biomedicine, the UNESCO Universal Declaration on the Human Genome and Human Rights, the requirements established in the Spanish legislation in the field of biomedical research, personal data protection and bioethics; as well as the provisions of the Law 14/2007, of July 3, of Biomedical Research; and RD 53/2013, of February 1. Renal function and diagnosis data were obtained from the patients' medical records. Renal function was monitored by means of $\mathrm{Cr}_{\mathrm{pl}}$, and AKI was defined and classified according to Kidney Disease: Improving Global Outcomes criteria [15]. Urine was collected upon admission to the Nephrology Department and was used to measure protein content (with a commercial kit from Bio-Rad, Madrid, Spain), N-acetyl-beta-D-glucosaminidase (NAG; with a commercial kit DZ062A-K from Diazyme Laboratories, Poway, CA, USA), PAI-1, and NGAL (as described below).

\section{Western Blot}

A volume of urine $(\mu \mathrm{L})$ from each rat corresponding to the same excretion fraction (i.e., same $\%$ of their daily urinary output) was separated by acrylamide electrophoresis. Proteins were transferred to an Immobilon-P Transfer Membrane (Millipore, Madrid, Spain) and incubated with primary antibodies against PAI-1 (BD Transduction Laboratories, CA, USA), followed by horseradish peroxidase-conjugated secondary antibodies and chemiluminescent detection (Immobilon Western Chemiluminescent HRP Substrate kit; Millipore, Madrid, Spain) with the ChemiDoc ${ }^{\mathrm{TM} M P}$ Imaging System (Bio-Rad, Madrid, Spain).

\section{ELISA}

PAI- 1 and NGAL were measured in human samples with the following commercial ELISAs: Human PAI-1 RAB0429 (Merck Life Science, Madrid, Spain) and Human NGAL ELISA Kit 036CE (BioPorto Diagnostics, Hellerup, Denmark) respectively, following the manufacturer's instructions.

\section{Statistical Analysis}

Data are represented as the mean \pm SEM of $n$ experiments, as indicated in each case. Animal's and patients' data normality was checked with the Shapiro-Wilk and Kolmogorov-Smirnov tests, respectively. Animal's non-parametric data were compared using Friedman test followed by the Dunn's post hoc test. For data with a normal distribution (animal's and patients'), a student $t$ test or a one-way ANOVA was used (Bonferroni post hoc tests were used for multiple comparisons). The GraphPad Prism 7.0 (GraphPad Software, San Diego, CA, USA) and the IBM SPSS Statistics 20 (IBM, Armonk, NY, USA) software were used. A $p$ value $<0.05$ was considered statistically significant.

\section{Results}

\section{PAI-1 Urinary Excretion Is Increased in Experimental}

\section{Models of Acute Tubular Necrosis}

The 3 AKI models used in this study (i.e., cisplatin and gentamicin nephrotoxicity and I/R injury) showed typical characteristics of intrinsic AKI, congruently with other studies carried out by us $[2,9,16]$ and others with these models. In all cases, rats showed increased $\mathrm{Cr}_{\mathrm{pl}}$ and urea concentrations and proteinuria compared to the controls (Fig. 1), and clear histological evidence of tubular necrosis (Fig. 2). Typical features of ATI, such as epithelial cell desquamation, tubular dilation, tubular necrosis, tubular obstruction, and infiltration, were detected mainly in the outer medulla of $\mathrm{I} / \mathrm{R}$ and cisplatin rats, and in the cortex of gentamicin rats. In the 3 models, the level of PAI- 1 increased, nearly in association with the progression and the extent of renal damage, in the urine, and in the renal tissue, as revealed by the tissue level of the PAI-1 protein and gene expression. However, PAI-1 was not increased in the plasma from the AKI models (Fig. 3).

\section{PAI-1 Is Produced by Damaged Kidneys and Secreted to the Urine Following AKI}

We next investigated the origin of UPAI-1. Specifically, we aimed at unraveling whether the PAI-1 was shed directly to the urine by renal cells, or it was filtered from the blood and not reabsorbed in the tubules. This is relevant from a diagnostic perspective, as the pathophysiological interpretation may vary with the source and mechanism leading to its increased urinary excretion. For this purpose, we used an in situ renal perfusion method that we had 


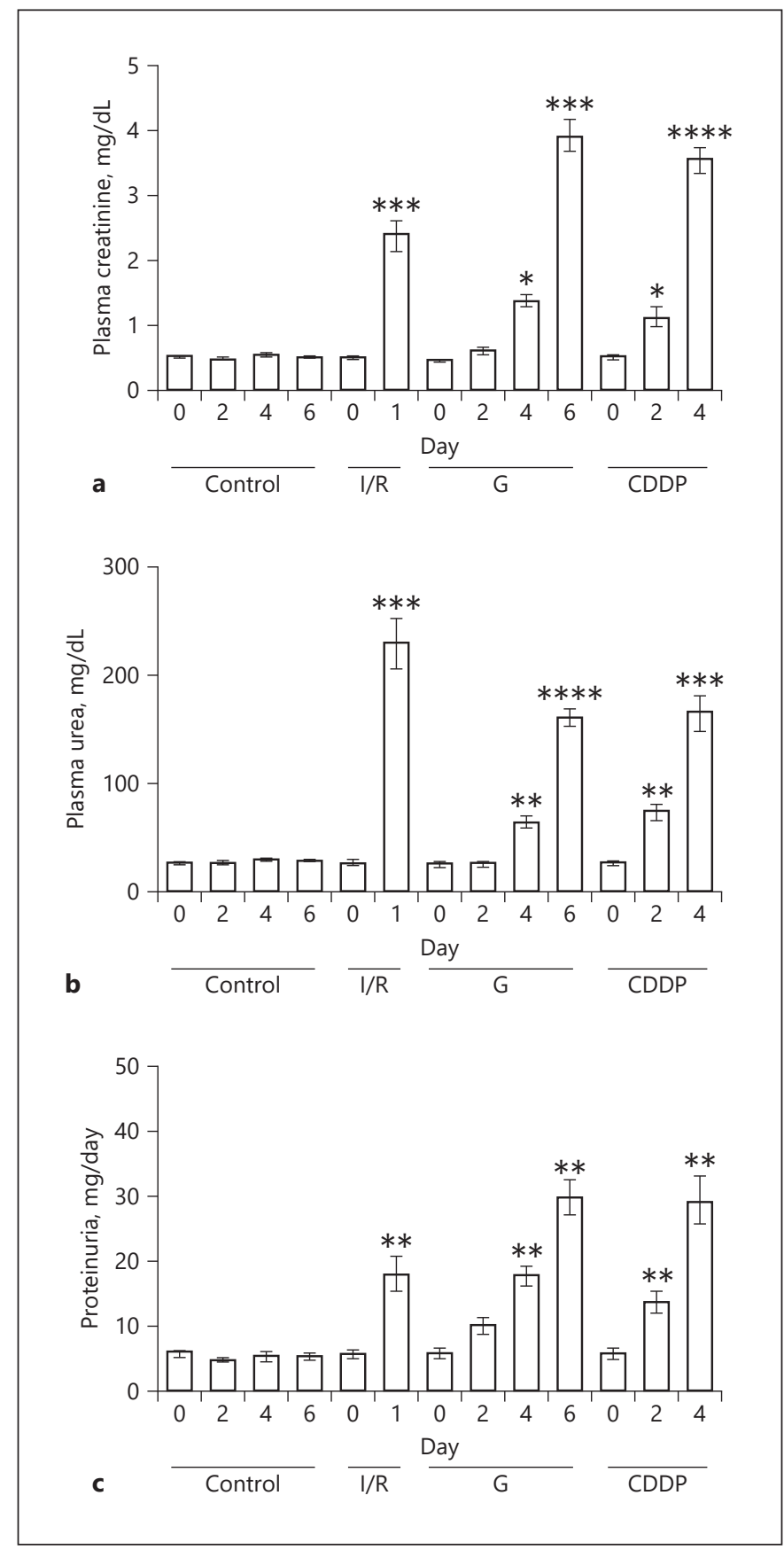

Fig. 1. Renal function assessment of the experimental models of AKI. a Plasma creatinine concentration; (b) plasma urea concentration; and (c) proteinuria. Data ( $n=6$ per group) are expressed as the mean \pm SEM. ${ }^{*} p<0.05,{ }^{* *} p<0.01,{ }^{* * *} p<0.001$, and ${ }^{* * * *} p<0.0001$ versus day 0 (basal) in the same group. Control, I/R, G, and CDDP. I/R, ischemia/reperfusion; G, gentamicin; CDDP, cisplatin.

previously used to study the origin of other biomarkers $[2,9,13]$. When the kidneys of control and AKI (induced by $I / R$ ) rats were perfused with a saline isotonic solution,
PAI-1 did not disappear from the urine of AKI rats (Fig. 4), as expected of a protein yielded directly to the urine by renal cells. Should PAI-1 have disappeared from the urine during perfusion, we would have concluded that it had a blood-born origin. It is concluded that because PAI-1 remained in the urine of AKI rats during perfusion, renal cells must have shed this protein directly to the urine. As a technical control, before perfusion with Krebs, the kidneys were perfused with blood from the same animal, by shunting a carotid artery with the renal artery by means of a catheter. In those circumstances, PAI-1 was still excreted with the urine in AKI rats, and not in controls (Fig. 4). We further investigated whether PAI-1 was also shed to the circulation from renal cells in AKI rats. As shown in Figure 4, the renal vein eluent of kidneys perfused with Krebs from AKI rats is not different from that in controls.

\section{uPAI-1 Increases in AKI Patients}

We analyzed the urinary excretion of PAI- 1 in patients with AKI. Results are shown as urinary concentration and also normalized to urinary creatinine or proteinuria. Urinary creatinine is a controversial but frequently used normalizing parameter [17]. Normalization by proteinuria suggests that PAI-1 excretion is not a general consequence of indiscriminate protein loss, but the reflection of a more specific phenomenon. In all cases, uPAI-1 is very low in controls. However, in AKI patients PAI-1 is markedly and statistically elevated (Fig. 5). As shown in Table 1, statistically significant but low correlations were found between the UPAI- 1 and those of NGAL and NAG, which suggests that the pathophysiological meaning conveyed by PAI- 1 is not redundant, but complementary, to the meaning of NGAL and NAG. Graphical depictions of these correlations are shown in online supplementary Fig. 1 (for all online suppl. material, see www.karger.com/doi/10.1159/000518455).

\section{Discussion}

The serine protease inhibitor (serpin) PAI-1 is the main inhibitor of tissue plasminogen activator and urokinase, the activators of plasminogen. Traditionally, the physiological and pathophysiological role of PAI-1 has been critically associated to the regulation of thrombolysis and hemostasis, as an inhibitor of fibrinolysis [18, 19], and its deficiency leads to hemorrhagic diathesis. PAI-1 is produced mainly by endothelial cells, but also by adipose tissue $[20,21]$. Various diseases, such as cancer, obesity, and the metabolic syndrome are related to higher levels of PAI-1, 
Fig. 2. Histological characterization of the experimental models of AKI. Representative images of the cortex and outer medullary area $(\times 400)$ from kidney specimens $(n=6$ per group) stained with hematoxylin and eosin from Control, I/R, G, and CDDP rats. Control rats show normal renal parenchyma (with intact tubules and glomeruli), whereas overt tubular necrosis is observed in the 3 models of AKI. Widespread evidence of tubular desepithelization (black arrows) and luminal tissue debris accumulation (asterisks) is seen most in the outer medullary region of the $I / R$ and cisplatin models, and in the cortex of the G model. $I / R$, ischemia/reperfusion; $G$, gentamicin; CDDP, cisplatin.

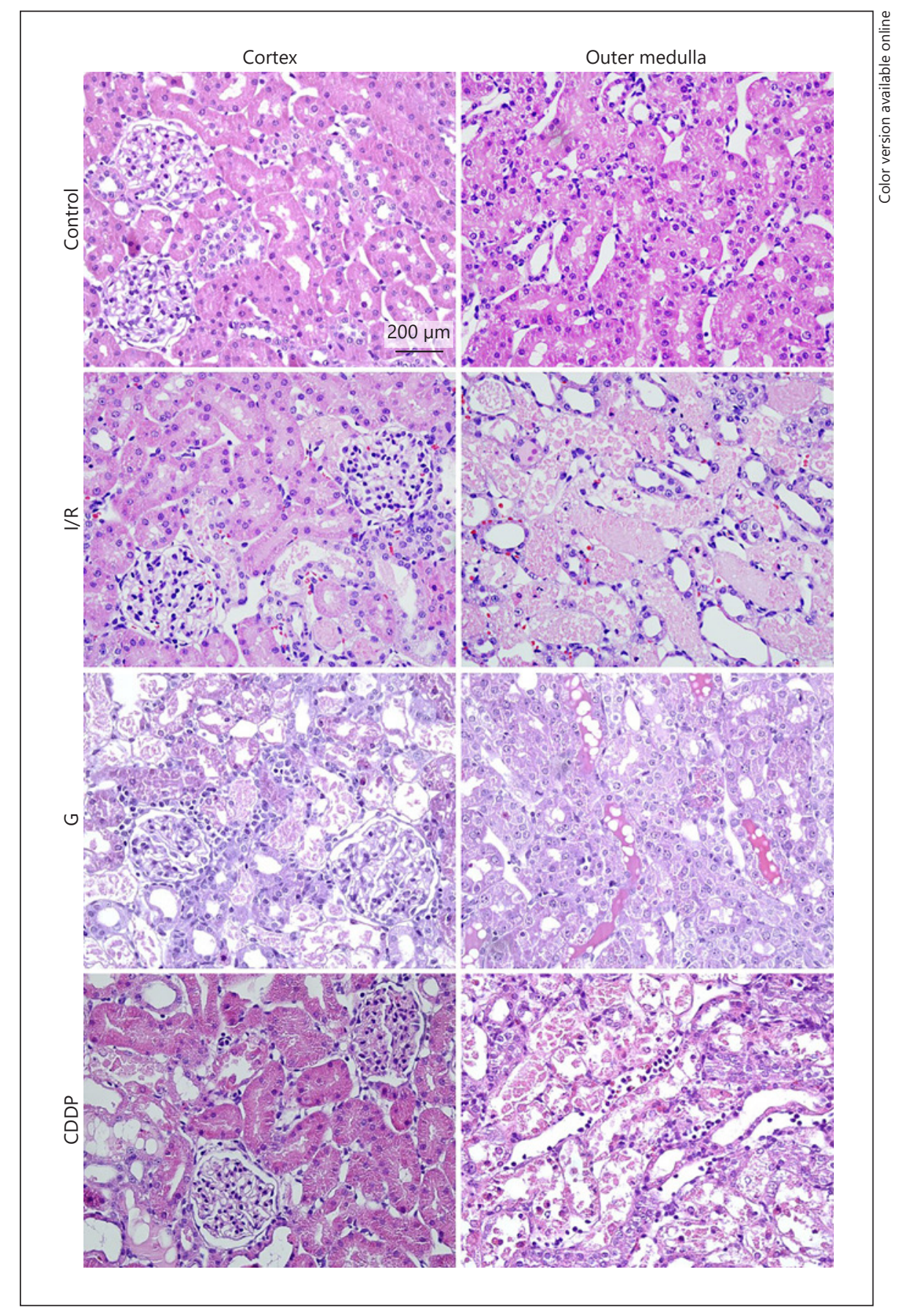

and increased thrombosis has been linked to these states [22]. PAI-1 also participates in the inflammatory process and has been found increased in damaged and inflamed tissues [23]. In the kidneys (and other organs), PAI-1 is a pro-fibrotic factor $[24,25]$ that inhibits plasmin formation, increases transforming growth factor $\beta$ expression, and stimulates fibroblasts and macrophage infiltration [26, 27]. Conversely, pharmacological inhibition of PAI-1 ameliorates fibrosis [28]. Specifically, PAI-1 plays an important role in renal fibrosis and progression of chronic kidney disease $[26,29,30]$. Of note, in disease states including fibrosis, tissue, and plasma levels of PAI-1 increase [29, 31], and uPAI- 1 also increases in specific conditions such as overt diabetic nephropathy [32] and active lupus nephritis [33]. 


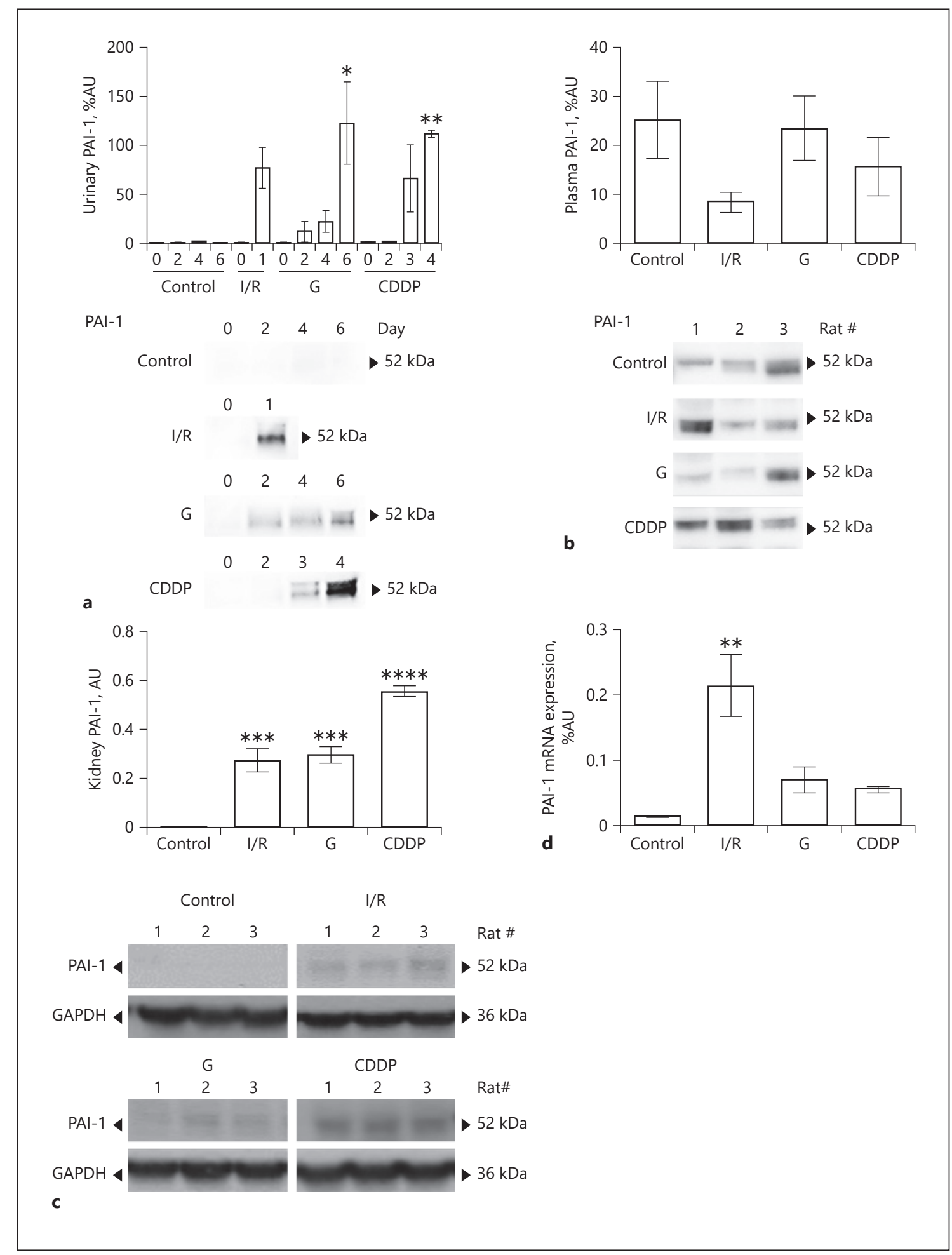

Fig. 3. PAI-1 was produced by the kidneys and increases in the urine during AKI. a Evolution of uPAI-1 levels. b Plasma PAI-1 levels at the end of the protocol. c Renal tissue PAI-1 levels. d PAI-1 renal gene expression. Data ( $n=3$ per group) are expressed as the mean \pm SEM. ${ }^{*} p<0.05$ and ${ }^{* *} p<0.01$ versus day 0 (basal) in the same group. ${ }^{* *} p<0.01,{ }^{* * *} p<0.001$ and ${ }^{* * * *} p<0.0001$ versus the control. Control, I/R, G, and CDDP. I/R, ischemia/ reperfusion; G, gentamicin; CDDP, cisplatin; uPAI-1, urinary PAI-1. 


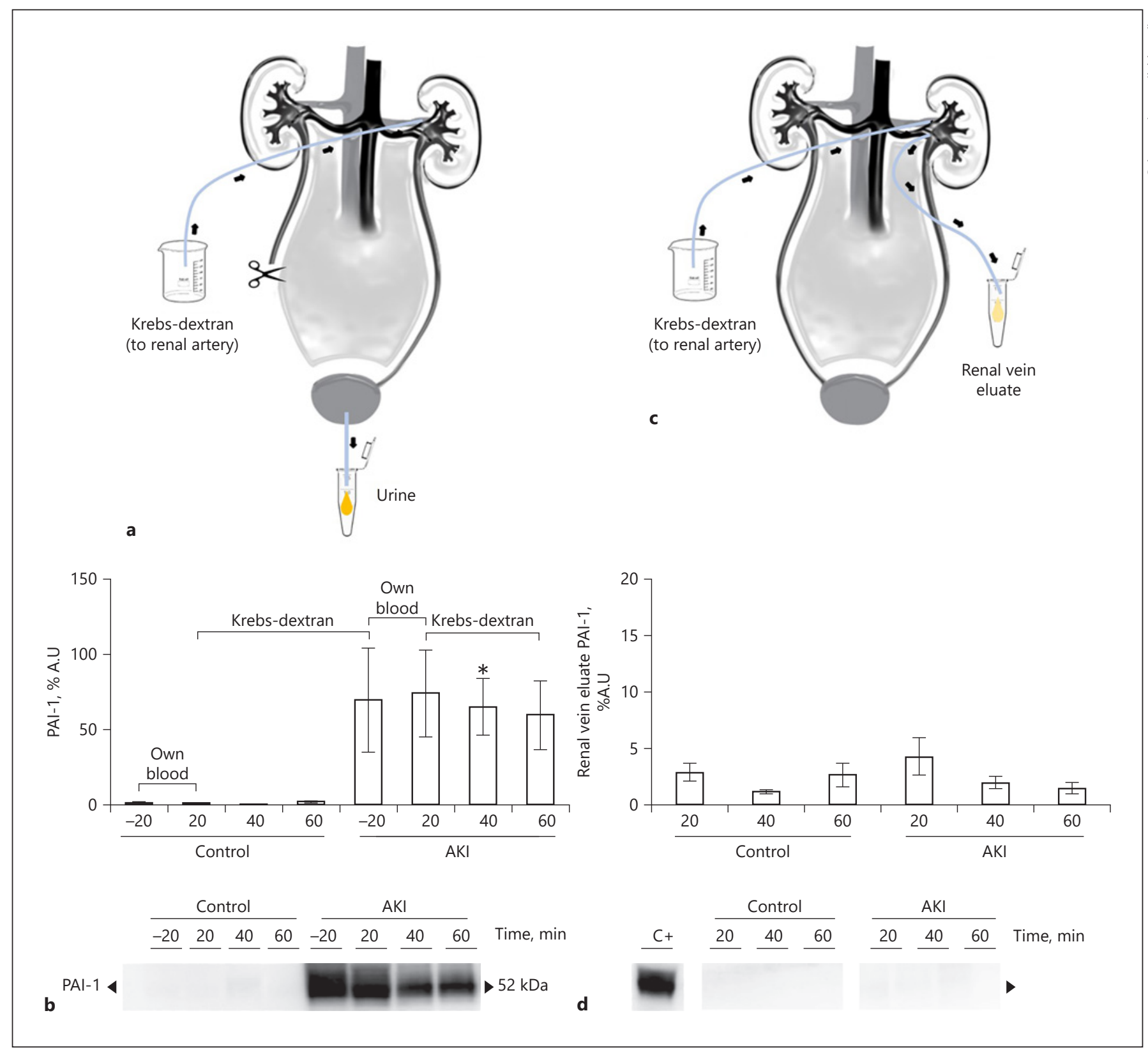

Fig. 4. PAI-1 was shed directly to the urine from renal cells. a Schematic representation of the experimental setup involving in situ kidney perfusion with Krebs-dextran solution or blood from the carotid artery, and urine collection from the bladder. b Representative image of Western blot analysis of UPAI-1 excretion during the 60-min perfusion with Krebs-dextran or own blood from the carotid artery (lower panel). Quantification of uPAI-1 excretion (upper panel). c Schematic representation of the experimental setup involving in situ kidney perfusion with Krebs-dextran solution, and effluent collection from the renal vein (upper panel). d Representative image of Western blot analysis of uPAI- 1 in the vein effluent during the 60-min perfusion with Krebs-dextran (lower panel). Quantification of PAI-1 in the effluent from the renal vein (upper panel). Data ( $n=3$ per group) are expressed as the mean \pm SEM. AU, arbitrary units. AKI, acute kidney injury; uPAI-1, urinary PAI-1.
PAI-1 has also been linked to AKI. PAI-1 participates in septic AKI [34] (a condition with an important inflammatory component [35]) and also in renal thrombotic microangi- opathies [36]. Plasma PAI-1 is increased in AKI patients in the context of acute lung injury [37], and in human lung transplantation-related AKI [38], which has been related 


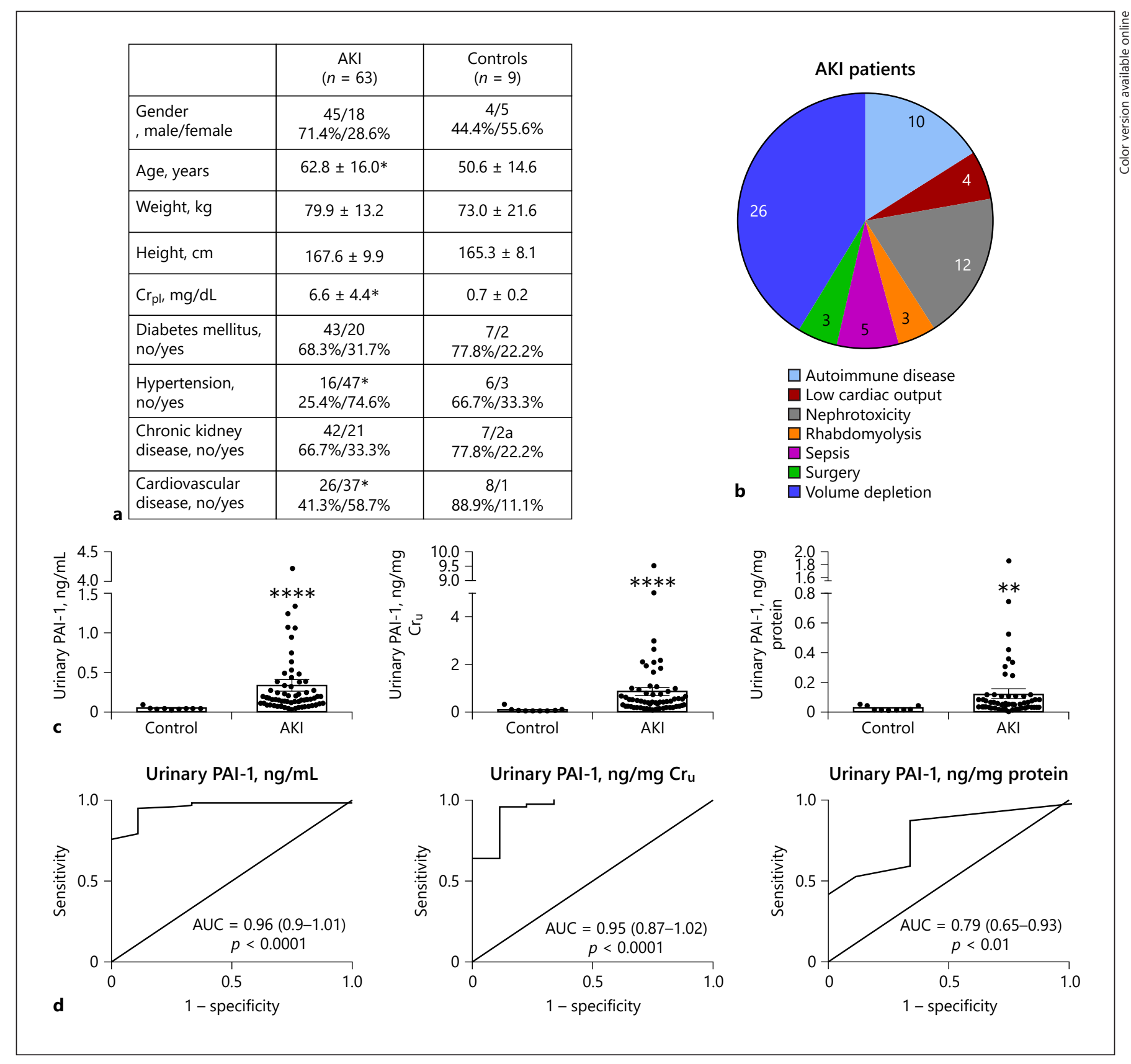

Fig. 5. uPAI-1 was increased in AKI patients. Patient characteristics (a) and AKI etiologies (b). c Urinary levels of PAI-1 in AKI patients and controls showing the mean \pm SEM of $n=9$ controls, and $n=63$ AKI patients. The left panel shows urinary concentration of PAI-1, whereas in the central and right panels PAI-1 concentration is normalized to urinary creatinine concentration, and to proteinuria, respectively. $\mathbf{d}$ ROC curves on the capacity of uPAI-
1 (left panel), uPAI-1 normalized to urinary creatinine (central panel), and uPAI-1 normalized to proteinuria (right panel). ${ }^{*} p<$ 0.05 versus control, ${ }^{* *} p<0.01$ versus control, and ${ }^{* * * *} p<0.0001$ versus control. AUC, area under the curve; $\mathrm{Cr}_{\mathrm{pl}}$, plasma creatinine concentration; $\mathrm{Cr}_{\mathrm{u}}$, urinary creatinine concentration; AKI, acute kidney injury; ROC, receiver operating characteristic; uPAI-1, urinary PAI-1. to renal microvascular injury $[38,39]$. PAI-1 is also increased in the urine of rats following toxic AKI induced by gentamicin [13] and iodinated contrast media [14]. Our present results show that in 3 etiologically different models of ATI (i.e., gentamicin and cisplatin nephrotoxicity and ischemic injury), PAI- 1 is overexpressed in the kidneys and 
Table 1. Results of the correlation analysis of uPAI-1 and other biomarkers of kidney injury

\begin{tabular}{|c|c|c|c|c|}
\hline & $\mathrm{Cr}_{\mathrm{pl}}, \mathrm{mg} / \mathrm{dL}$ & Proteinuria, mg/mL & uNGAL, ng/mL & $\mathrm{uNAG}, \mathrm{IU} / \mathrm{mL}$ \\
\hline \multirow[t]{2}{*}{ uPAI-1, ng/mL } & 0.116 & 0.094 & $0.309^{*}$ & 0.199 \\
\hline & $\mathrm{Cr}_{\mathrm{pl}}, \mathrm{mg} / \mathrm{dL}$ & $\begin{array}{l}\text { proteinuria, } \\
\mathrm{mg} / \mathrm{mg} \mathrm{Cr}_{\mathrm{u}}\end{array}$ & $\begin{array}{l}\mathrm{uNGAL} \\
\mathrm{ng} / \mathrm{mg} \mathrm{Cr}_{\mathrm{u}}\end{array}$ & $\begin{array}{l}\mathrm{uNAG} \\
\mathrm{IU} / \mathrm{mg} \mathrm{Cr}_{\mathrm{u}}\end{array}$ \\
\hline \multirow[t]{2}{*}{$\mathrm{uPAI}-1, \mathrm{ng} / \mathrm{mg} \mathrm{Cr}_{\mathrm{u}}$} & 0.154 & $0.309^{*}$ & $0.426^{* * *}$ & $0.374^{* *}$ \\
\hline & $\mathrm{Cr}_{\mathrm{pl}}, \mathrm{mg} / \mathrm{dL}$ & & $\begin{array}{l}\text { uNGAL, } \\
\text { ng/mg protein }\end{array}$ & $\begin{array}{l}\text { uNAG, } \\
\text { IU/mg protein }\end{array}$ \\
\hline uPAI-1, ng/mg protein & 0.154 & & $0.378^{* *}$ & $0.363^{* *}$ \\
\hline
\end{tabular}

In the upper panel, the study was carried out with raw data from urinary biomarkers. In the central panel, urinary biomarkers were normalized to urinary creatinine concentration $\left(\mathrm{Cr}_{\mathrm{u}}\right)$. In the lower panel, biomarkers were normalized to proteinuria. Data are expressed as Spearman's correlation coefficient $(\rho)$. $\mathrm{Cr}_{\mathrm{pl}}$, plasma creatinine concentration; Prot, proteinuria; $\mathrm{Cr}_{\mathrm{u}}$, urinary creatinine concentration; IU, international units; $\mathrm{uNAG}$, urinary NAG activity; uNGAL, urinary NGAL concentration; uPAI-1, urinary PAI-1. ${ }^{*} p<0.05{ }^{* *} p<0.01$. $* * * p<0.001$.

its urinary excretion is increased. In these ATI models, as in those reported in $[13,14]$, inflammation amplifies tubular damage and contributes to reducing glomerular filtration rate [40-42], the hallmark alterations of acute tubular disease. It is thus possible (and likely) that PAI-1 be involved in these inflammatory responses.

Pathological coagulation might also explain PAI-1 participation in toxic and ischemic AKI. Renal pro-coagulation events have been reported for these types of AKI, including endothelial dysfunction [43, 44], expression of adhesion molecules in platelets [45] and endothelial cells [43], and fibrin deposition in the vascular lumen, the glomerulus, Bowman's capsule and interstitium [46, 47]. In agreement, anticoagulation reduces tubular obstruction, probably by preventing conversion of filtered fibrinogen into fibrin $[47,48]$. These pro-coagulant events do not seem to lead to full thrombotic processes, but rather to microvascular congestion $[43,44]$ resulting from blood cells and platelet aggregation to the endothelium [43, 49]. Endothelial dysfunction also enables white cell infiltration, diapedesis, vascular hyper-permeability, and inflammation [50]. In turn, inflammation contracts renal vessels, reduces renal blood flow and glomerular filtration rate [40, 41], and recruits pro-inflammatory and pro-coagulant endothelial signaling [51], which closes a vicious feedback cycle between inflammation, endothelial dysfunction, reduced renal flow, and glomerular filtration rate.
Our results also indicate that, in our ischemic ATI model, PAI-1 may be elevated in the urine by direct secretion from renal cells. Hypothetically, this secretion may be the consequence of the pro-coagulation and inflammatory scenario described above. Thus, PAI-1 is one of the few biomarkers, along with tail-less complex polypeptide-1 eta subunit (TCP1-eta) [2,52], for which direct shedding from renal cells has been experimentally supported, in association with AKI. In fact, it has been generally assumed that other biomarkers increased in the renal tissue and urine following AKI, were directly shed to the urine from damaged renal structures. However, recent studies have shown differently, as described above for NGAL, TIMP-2, and IGFBP. Accordingly, uPAI-1 might be a direct pathophysiological indicator of renal inflammatory status. Although the diagnostic information conveyed by uPAI- 1 needs to be further explored, this marker provides differential and additive diagnostic value.

The established knowledge indicates that PAI-1 might not be a general parenchymal damage level reporter, but a biomarker of a more specific pathological event, such as endothelium-mediated inflammatory damage. In agreement, inflammation is an important pathogenetic factor in AKI [40, 41, 51, 53]. Interestingly, the low correlation found with the level of NGAL and NAG implies that the type of damage or pathophysiological events causing NGAL and NAG excretion in these patients is different from the damage leading to the excretion of PAI-1. This level of biomarker specificity is needed for the sought 
pathophysiological diagnosis of AKI, and for prognosis estimation based on the underlying individual damage pattern $[2,5]$. Increasing knowledge of pathophysiological granularity provided by biomarker-based liquid autopsies should thus allow a more precise patient stratification and diagnosis, irrespective of etiology. The challenge for the immediate future is to identify the specific damage patterns, or pathological mechanisms etiologically associated to each biomarker.

\section{Conclusions}

In this article, we further characterize PAI-1, a regulator of coagulation, as a urinary biomarker of AKI, hitherto vaguely associated to this syndrome. Most importantly, we show that the PAI-1 detected in the urine following AKI is produced by damaged kidneys and shed directly to the urine. uPAI-1 is thus a direct probe of a specific pathological event of renal injury, which needs to be further explored. UPAI- 1 is one of the few AKI biomarkers with substantiated renal origin, and thus a potentially valuable tool for a prospective pathophysiological diagnostic panel.

\section{Statement of Ethics}

All clinical protocols were approved by the Hospital Universitario Marqués de Valdecilla (Santander, Spain) Ethics Committee and were conducted according to the principles established in the Declaration of Helsinki (World Medical Assembly), the Council of Europe Convention on Human Rights and Biomedicine, the UNESCO Universal Declaration on the Human Genome and $\mathrm{Hu}$ man Rights, the requirements established in the Spanish legislation in the field of biomedical research, personal data protection and bioethics; as well as the provisions of the Law 14/2007, of July 3, of Biomedical Research; and RD 53/2013, of February 1. Volunteer patients provided written consent. All procedures involving experimental animals were approved by the Bioethics Committee of the University of Salamanca. Animals were handled according to the guidelines of the European Community Council Directive 2010/63/UE and to the current Spanish legislation for experimental animal use and care, RD 53/2013.

\section{Conflict of Interest Statement}

The authors declare that they have no competing interests.

\section{Funding Sources}

This study was supported by Grants from the Instituto de Salud Carlos III (DT15S/00166, PI10-00624, PI15/01055, PI18/ 00996 and Retic RD016/0009/0025, REDINREN), and FEDER funds.

\section{Author Contributions}

M.P.-S., Y.Q., A.G.C., V.B.-G., and S.M.S.-N. carried out the experiments with animal models and the biochemical studies. C.A.-B., A.B.-H., M.A.R.-B., C.G.-A., and M.A.R.-B. designed and conducted the clinical study. A.G.C., M.P.-S., and S.M.S.-M. performed statistical data analysis and plotted the graphics. S.M.S.-M. and F.J.L.-H. designed and coordinated the overall study. F.J.L.-H. drafted the document.

\section{Data Availability Statement}

The datasets used and/or analyzed during the current study are available from the corresponding author on reasonable request.

\section{References}

1 Thomas ME, Blaine C, Dawnay A, Devonald MA, Ftouh S, Laing C, et al. The definition of acute kidney injury and its use in practice. Kidney Int. 2015;87:62-73.

2 Sancho-Martínez SM, Sánchez-Juanes F, Blanco-Gozalo V, Fontecha-Barriuso M, Prieto-García L, Fuentes-Calvo I, et al. Urinary TCP1-eta: a cortical damage marker for the pathophysiological diagnosis and prognosis of acute kidney injury. Toxicol Sci. $2020 \mathrm{Mar}$ $1 ; 174(1): 3-5$

3 Belayev LY, Palevsky PM. The link between acute kidney injury and chronic kidney disease. Curr Opin Nephrol Hypertens. 2014;23: 149-54.

4 Canaud G, Bonventre JV. Cell cycle arrest and the evolution of chronic kidney disease from acute kidney injury. Nephrol Dial Transplant. 2015 Apr [cited 2020 Feb 4];30(4):575-83.

5 Moledina DG, Parikh CR. Phenotyping of acute kidney injury: beyond serum creatinine. Semin Nephrol. 2018;38:3-11.

6 Kellum JA, Chawla LS. Cell-cycle arrest and acute kidney injury: the light and the dark sides. Nephrol Dial Transplant. 2016;31(1):16-22.

7 Ronco C. Acute kidney injury: from clinical to molecular diagnosis. Crit Care. 2016;20(1):201.

8 Ronco C, Kellum JA, Haase M. Subclinical AKI is still AKI. Crit Care. 2012 Jun 21 [cited 2020 Feb 4];16(3):313.

9 Sancho-Martínez SM, Blanco-Gozalo V, Quiros Y, Prieto-García L, Montero-Gómez MJ, Docherty NG, et al. Impaired tubular reabsorption is the main mechanism explaining increases in urinary NGAL excretion following acute kidney injury in rats. Toxicol Sci. 2020 May 1;175(1):75-86.

10 Skrypnyk NI, Gist KM, Okamura K, Montford JR, You Z, Yang H, et al. IL-6-mediated hepatocyte production is the primary source of plasma and urine neutrophil gelatinase-associated lipocalin during acute kidney injury. Kidney Int. 2020 May 1;97(5):966-79.

11 Johnson ACM, Zager RA. Mechanisms underlying increased TIMP2 and IGFBP7 urinary excretion in experimental AKI. J Am Soc Nephrol. 2018 Aug 1;29(8):2157-67.

12 Iwaki T, Urano T, Umemura K. PAI-1, progress in understanding the clinical problem and its aetiology. Br J Haematol. 2012 [ cited 2020 Oct 12];157:291-8. 
13 Quiros Y, Ferreira L, Sancho-Martínez SM, González-Buitrago JM, López-Novoa JM, López-Hernández FJ. Sub-nephrotoxic doses of gentamicin predispose animals to developing acute kidney injury and to excrete ganglioside M2 activator protein. Kidney Int. 2010 Nov;78(10):1006-15.

14 Quiros Y, Sánchez-González PD, LópezHernández FJ, Morales AI, López-Novoa JM. Cardiotrophin-1 administration prevents the renal toxicity of iodinated contrast media in rats. Toxicol Sci. 2013 Apr;132(2): 493-501.

15 Khwaja A. KDIGO clinical practice guidelines for acute kidney injury. Nephron Clin Pract. 2012;120:c179-84.

16 Casanova AG, Vicente-Vicente L, HernándezSánchez MT, Prieto M, Rihuete MI, Ramis LM, et al. Urinary transferrin pre-emptively identifies the risk of renal damage posed by subclinical tubular alterations. Biomed Pharmacother. 2020 Jan 1 [cited 2020 Feb 16];121: 109684.

17 Waikar SS, Sabbisetti VS, Bonventre JV. Normalization of urinary biomarkers to creatinine during changes in glomerular filtration rate. Kidney Int. 2010;78(5):486-94.

18 Iwaki T, Urano T, Umemura K. PAI-1, progress in understanding the clinical problem and its aetiology. Br J Haematol. 2012;157:291-8.

19 Yasar Yildiz S, Kuru P, Toksoy Oner E, Agirbasli M. Functional stability of plasminogen activator inhibitor-1. ScientificWorldJournal. 2014;2014:1.

20 Schäfer K, Konstantinides S. Adipokines and thrombosis. Clin Exp Pharmacol Physiol. 2011 Dec;38(12):864-71.

$21 \mathrm{Kaji} \mathrm{H}$. Adipose tissue-derived plasminogen activator inhibitor-1 function and regulation. Compr Physiol. 2016 Oct 1;6(4):1873-96.

22 Wood PA. Connecting the dots: obesity, fatty acids and cancer. Lab Invest. 2009;89:1192-4.

23 Renckens R, Roelofs JJ, De Waard V, Florquin $\mathrm{S}$, Lijnen HR, Carmeliet $\mathrm{P}$, et al. The role of plasminogen activator inhibitor type 1 in the inflammatory response to local tissue injury. J Thromb Haemost. 2005 May [cited 2020 Oct 12];3(5):1018-25.

24 Ghosh AK, Vaughan DE. PAI-1 in tissue fibrosis. J Cell Physiol. 2012;227:493-507.

25 Rabieian R, Boshtam M, Zareei M, Kouhpayeh S, Masoudifar A, Mirzaei H. Plasminogen activator inhibitor type- 1 as a regulator of fibrosis. J Cell Biochem. 2018 Jan 1;119(1):17-27.

26 Huang Y, Noble NA. PAI-1 as a target in kidney disease. Curr Drug Targets. 2007 Aug 28; 8(9):1007-15

27 Seo JY, Park J, Yu MR, Kim YS, Ha H, Lee HB. Positive feedback loop between plasminogen activator inhibitor- 1 and transforming growth factor-beta1 during renal fibrosis in diabetes. Am J Nephrol. 2009 Dec;30(6):481-90.
28 Jeong BY, Uddin MJ, Park JH, Lee JH, Lee HB, Miyata T, et al. Novel plasminogen activator inhibitor-1 inhibitors prevent diabetic kidney injury in a mouse model. PLoS One. 2016 Jun 1;11(6):e0157012.

29 Ma LJ, Fogo AB. PAI- 1 and kidney fibrosis. Front Biosci (Landmark Ed). 2009 Jan 1;14(6): 2028-41.

30 MaŁgorzewicz S, Skrzypczak-Jankun E, Jankun J. Plasminogen activator inhibitor-1 in kidney pathology (review). Int J Mol Med. 2013 Mar; 31(3):503-10.

$31 \mathrm{Ha} \mathrm{H}, \mathrm{Oh} \mathrm{EY}$, Lee HB. The role of plasminogen activator inhibitor 1 in renal and cardiovascular diseases. Nat Rev Nephrol. 2009;5: 203-11.

32 Verhave JC, Bouchard J, Goupil R, Pichette V, Brachemi S, Madore F, et al. Clinical value of inflammatory urinary biomarkers in overt diabetic nephropathy: a prospective study. Diabetes Res Clin Pract. 2013 Sep;101(3): 333-40.

33 Landolt-Marticorena C, Prokopec SD, Morrison $\mathrm{S}$, Noamani B, Bonilla $\mathrm{D}$, Reich $\mathrm{H}$, et al. A discrete cluster of urinary biomarkers discriminates between active systemic lupus erythematosus patients with and without glomerulonephritis. Arthritis Res Ther. 2016 Oct $4 ; 18(1): 218$

34 Gupta KK, Donahue DL, Sandoval-Cooper MJ, Castellino FJ, Ploplis VA. Abrogation of plasminogen activator inhibitor-1-vitronectin interaction ameliorates acute kidney injury in murine endotoxemia. PLoS One. 2015 Mar 23 [cited 2020 Oct 12];10(3):e0120728.

35 Gomez H, Ince C, De Backer D, Pickkers P, Payen D, Hotchkiss J, et al. A unified theory of sepsis-induced acute kidney injury: Inflammation, microcirculatory dysfunction, bioenergetics, and the tubular cell adaptation to injury. Shock. 2014 Jan [cited 2020 Oct 12]; 41(1):3-11.

36 Eddy AA. Plasminogen activator inhibitor-1 and the kidney. Am J Physiol Renal Physiol. 2002 [cited 2020 Oct 12];283:F209-20.

37 Liu KD, Glidden DV, Eisner MD, Parsons PE, Ware LB, Wheeler A, et al. Predictive and pathogenetic value of plasma biomarkers for acute kidney injury in patients with acute lung injury. Crit Care Med. 2007;35(12):2755.

38 Forker CM, Miano TA, Reilly JP, Oyster ML, Porteous MK, Cantu EE, et al. Postreperfusion plasma endothelial activation markers are associated with acute kidney injury after lung transplantation. Am J Transplant. 2019 Aug $1 ; 19(8): 2366-73$.

39 Molitoris BA. Therapeutic translation in acute kidney injury: the epithelial/endothelial axis. J Clin Invest. 2014 Jun [cited 2020 Feb 3]; 124(6):2355-63.

40 Lopez-Novoa JM, Quiros Y, Vicente L, Morales AI, Lopez-Hernandez FJ. New insights into the mechanism of aminoglycoside nephrotoxicity: an integrative point of view. Kidney Int. 2011 Jan [cited 2020 Oct 12];79(1):3345.

41 Sánchez-González PD, López-Hernández FJ, López-Novoa JM, Morales AI. An integrative view of the pathophysiological events leading to cisplatin nephrotoxicity. Crit Rev Toxicol. 2011 [cited 2020 Oct 12];41:803-21.

42 Sharfuddin AA, Molitoris BA. Pathophysiology of ischemic acute kidney injury. Nat Rev Nephrol. 2011 [cited 2020 Oct 12];7:189-200.

43 Devarajan P. Update on mechanisms of ischemic acute kidney injury. J Am Soc Nephrol. 2006;17:1503-20.

44 Basile DP, Anderson MD, Sutton TA. Pathophysiology of acute kidney injury. Compr Physiol. 2012 Apr [cited 2020 Oct 12];2(2): 1303-53.

45 Singbartl K, Forlow SB, Ley K. Platelet, but not endothelial, P-selectin is critical for neutrophilmediated acute postischemic renal failure. FASEB J. 2001 Nov [cited 2020 Oct 12];15(13): 2337-44.

46 Loverre A, Ditonno P, Crovace A, Gesualdo L, Ranieri E, Pontrelli P, et al. Ischemia-reperfusion induces glomerular and tubular activation of proinflammatory and antiapoptotic pathways: differential modulation by rapamycin. J Am Soc Nephrol. 2004 Oct [cited 2020 Oct 12];15(10):2675-86.

47 Enestrom Druid SH, Rammer L. Fibrin deposition in the kidney in post-ischaemic renal damage. Br J Exp Pathol. 1988 Jun [cited 2020 Oct 12];69(3):387-94.

48 Druid H, Eneström S, Rammer L. Effect of anticoagulation upon nephron obstruction in experimental acute ischaemic renal failure. A morphological study. Int J Exp Pathol. 1998 [cited 2020 Oct 12];79(1):55-66.

49 Molitoris BA, Sutton TA. Endothelial injury and dysfunction: role in the extension phase of acute renal failure. Kidney Int. 2004;66: 496-9.

50 Ozden A, Sarioglu A, Demirkan NC, Bilgihan A, Düzcan E. Antithrombin III reduces renal ischemia-reperfusion injury in rats. Res Exp Med. 2001;200:195-203.

51 Bonventre JV. Pathophysiology of acute kidney injury: roles of potential inhibitors of inflammation. Contrib Nephrol. 2007 [cited 2020 Dec 19];156:39-46.

52 Blanco-Gozalo V, Casanova AG, SanchoMartínez SM, Prieto M, Quiros Y, Morales AI, et al. Combined use of GM2AP and TCP1-eta urinary levels predicts recovery from intrinsic acute kidney injury. Sci Rep. 2020 Dec 1; 10(1):11599.

53 Mulay SR, Holderied A, Kumar SV, Anders HJ. Targeting inflammation in so-called acute kidney injury. Semin Nephrol. 2016 [cited 2020 Dec 19];36:17-30. 